\title{
PERIÓDICOS CIENTÍFICOS BRASILEIROS A PARTIR DE SEUS INDEXADORES: CENÁRIO ATUAL E PERSPECTIVAS FUTURAS PARA A ADMINISTRAÇÃO, CONTABILIDADE E TURISMO
}

\author{
Rodrigo Assunção Rosa ${ }^{1}$; Marcello Romani-Dias²
}

\author{
Como citar: \\ ROSA, Rodrigo Assunção; ROMANI-DIAS, Marcello. Periódicos científicos brasileiros a partir de seus \\ indexadores:cenário atual e perspectivas futuras para a administração, contabilidade e turismo. In: ABEC \\ MEETING, 1, 2017, Curitiba. Anais... Curitiba: Associação Brasileira de Editores Científicos, 2017. p. $37-44$. \\ http://dx.doi.org/10.21452/abecmeeting.2017.007
}

\begin{abstract}
Resumo: A inserção de periódicos científicos em indexadores de relevância tem representado papel fundamental na disseminação e na qualidade da publicação científica de diversas áreas. Todavia, esta discussão tem sido negligenciada no campo de Administração, Contabilidade e Turismo. A partir do levantamento da população de 304 periódicos brasileiros do campo, o objetivo deste estudo é o de analisar o cenário destes periódicos a partir de seus principais indexadores. Demonstramos que os periódicos brasileiros ainda não atingiram presença significativa nos principais indexadores científicos, principalmente nos mais consolidados, tais como Web of Science, Scopus e Scielo. Identificamos que os periódicos estão inseridos em uma elevada quantidade de indexadores, todavia, esta quantidade não confere qualidade, mas sim, tal qualidade pode ser verificada a partir da inclusão destes periódicos em indexadores que são centrais para a comunidade científica. Diante destes resultados, discutimos as principais implicações deste cenário para a publicação científica do campo.
\end{abstract}

Palavras-Chave: Publicação Científica; Periódicos Científicos; Indexadores; Qualidade.

\begin{abstract}
The inclusion of scientific journals in relevance indexes has played a fundamental role in the dissemination and quality of scientific publication in several areas. However, this discussion has been neglected in the field of Administration, Accounting and Tourism. From the survey of 304 brazilian journals in the field, the objective of this study is to analyze the scenario of these journals from their central indexes. We have shown that the brazilian journals have not yet reached a significant presence in the main indexes, especially in the most consolidated ones, such as Web of Science, Scopus and Scielo. We identified that the journals are inserted in a high number of indexes, however, this quantity does not confer quality, but rather, such quality can be verified from the inclusion of these journals in indexers that are central to the scientific community. Given these results, we discuss the main implications of this scenario for the scientific publication of the field.
\end{abstract}

Keywords: Scientific Publishing; Scientific journals; Indexers; Quality.

\section{CONTEXTO E OBJETIVO}

Ao longo da última década o notável crescimento do campo científico de Administração, Contabilidade e Turismo no Brasil tem sido acompanhado por reflexões importantes no que diz respeito ao seu modo de produção e reprodução (Bertero et al., 2013). Neste amplo e complexo arcabouço de ideias, uma das preocupações centrais da comunidade acadêmica está relacionada as práticas de publicação científica e, mais especificamente, de periódicos científicos (Alcadipani, 2017; Diniz, 2017; Farias, 2017; Trzesniak, 2017).

Dentro da discussão sobre periódicos científicos, destacam-se a análise das práticas de gestão editorial (Sandes-Guimarães \& Diniz, 2014; Araújo et al., 2017), a discussão sobre os rankings e qualidade dos periódicos (Oliveira, Cendón e Cirino, 2017), e a internacionalização destes sob diferentes perspectivas (Kimura et al., 2014; Alcadipani, 2017; Diniz, 2017; Farias, 2017). Estes aspectos têm levado a avanços consideráveis no aumento da 
qualidade e rigor do campo. Apesar disso, ainda são necessários esforços para o aumento da qualidade de nossa produção e para a clareza de propósito e disseminação internacional da pesquisa por meio dos periódicos (Kimura et al., 2014; Diniz, 2017; Farias, 2017).

Neste contexto surge o papel dos indexadores científicos, responsáveis por reunirem um conjunto de títulos de periódicos em suas bases de dados e de darem visibilidade às publicações científicas tanto em âmbito nacional quanto internacional (Collazo-Reyes, 2015). Apesar de os principais indexadores terem um papel fundamental na disseminação e qualidade dos periódicos e das publicações científicas, sua relevância para a produção científica no campo de Administração, Contabilidade e Turismo tem sido negligenciada pelos estudos acadêmicos. Diante desta lacuna, propomos a seguinte questão de pesquisa: A partir de seus principais indexadores, qual é o cenário dos periódicos científicos brasileiros, e com base em nossa análise, que implicações este cenário traz para a publicação científica do campo?

\section{INDEXADORES NO CONTEXTO DA PUBLICAÇÃO CIENTÍFICA}

Oliveira et al. (2017) ao revisarem a literatura científica nacional e internacional sobre modelos para avaliação da qualidade de periódicos científicos identificaram que a indexação é um dos critérios mais presentes em pesquisas que abordam este tema. A entrada e a continuidade em indexadores de relevância permite que os periódicos revejam constantemente suas práticas e processos, visto que parte essencial destes indexadores exige uma série de rígidos critérios de qualidade para que os periódicos sejam indexados, e também para que permaneçam no indexador (Rozemblum et al. 2015).

Ao realizar a revisão de pesquisas científicas em âmbito nacional e internacional a respeito dos indexadores científicos identificamos dez principais: Google Scholar, Web of Science, Scopus, Scielo, Spell, Ebsco, Proquest, Doaj, Latindex e Redalyc (Falagas et al., 2008; Campanário \& Santos, 2011; Blattmann \& Santos,2014; Cetto \& Alonso-Gamboa, 2014; Calvert, 2015; Amaro, Harzing \& Alakangas, 2015; Silva \& Carvalho, 2016; Harzing \& Alakangas, 2015; Diniz, 2017; Trzesniak, 2017).

A inclusão dos periódicos científicos nestes indexadores permite à ciência o aumento do rigor e da qualidade e o distanciamento das publicações de baixo nível e de baixo impacto. Os critérios de boas práticas exigidos pelos indexadores tiram os periódicos científicos de seu status quo e influenciam tanto a dimensão científica quanto a dimensão administrativa dos periódicos (Sandes-Guimarães, \& Diniz, 2014). Rozemblum et al. (2015) ao analisarem diferentes tipos de indexadores, identificaram diversos indicadores relacionados à qualidade 
editorial, como aspectos relacionados à gestão e questões formais, de conteúdo, como por exemplo, originalidade e revisão por pares, e de visibilidade, tais como o acesso, as relações institucionais, a indexação e o prestígio do periódico.

Por fim, a internacionalização dos periódicos científicos, por meio dos indexadores científicos, tem sido uma das preocupações recorrentes de pesquisadores do campo (Alcadipani, 2017; Farias, 2017; Diniz, 2017; Kimura et. al 2014). Como exemplo, CollazoReyes (2015) demonstra que o cenário de indexação dos periódicos latino-americanos em bases científicas internacionais é expressivo, tendo o Brasil ganhado destaque, por meio do aumento de novos periódicos indexados. Todavia, o crescimento do número de periódicos indexados não tem acompanhado o aumento da internacionalização da publicação de artigos em âmbito mundial, principalmente no campo de Administração, Contabilidade e Turismo, em que ainda são necessários esforços para que os periódicos, em conjunto, atinjam um maior nível de integração dentro dos principais indexadores científicos nacionais e internacionais (Diniz, 2017).

\section{SÍNTESE METODOLÓGICA}

Haja visto que não existe um indexador que contemple todos os periódicos científicos presentes no campo, consideramos a utilização da classificação do Quadriênio (2013-2016) realizada pelo sistema Qualis-Periódicos publicado pela Coordenação de Aperfeiçoamento de Pessoal de Nível Superior (Capes) para nosso critério de seleção. Portanto, no decorrer do segundo semestre de 2017, para a definição da população de periódicos científicos nacionais do levantamento, procedemos inicialmente com a exclusão do estrato A1 (por conter apenas periódicos com origem fora do Brasil) e de periódicos de caráter técnico ou estritamente aplicado (estrato C). Em seguida, fomos aos websites dos periódicos presentes entre os estratos A2 a B5 e realizamos a análise de seus focos e escopos. Destaca-se que dentre os estratos analisados excluímos de nossa base: (i) periódicos que estavam replicados na lista e periódicos impressos; (ii) periódicos originários de outros países; (iii) periódicos em que o foco e escopo estão fora do campo definido neste estudo, ou seja periódicos multidisciplinares ou interdisciplinares que não tenham como centrais os temas do campo e periódicos de outras áreas do conhecimento e (iv) periódicos de acesso privado (pagos).

A partir desta sistematização chegamos a um conjunto de 304 periódicos científicos brasileiros correspondentes à população do campo de Administração, Contabilidade e Turismo, que representam $8,53 \%$ do total (3562) de revistas contidas na lista inicial do 
Qualis-Periódicos para a área. Das 304 revistas selecionadas para a análise 17 (5,59\%) estão no estrato A2, $30(9,87 \%)$ no B1, $42(13,82 \%)$ no B2, 70 (23,03\%) no B3, $107(35,20 \%)$ no B4 e 38 (12,50\%) no B5. Realizamos o levantamento dos principais indexadores mencionados pelos periódicos em seus websites e, em seguida, para a triangulação dos dados referentes aos indexadores informados nos websites dos periódicos, realizamos uma sistemática checagem nos dez principais indexadores apontados pela literatura como sendo os mais relevantes no contexto da produção científica.

\section{PRINCIPAIS RESULTADOS ENCONTRADOS}

A Tabela 1 trata da relação entre os dez principais indexadores científicos com frequência de periódicos presentes nos estratos do Qualis-Periódicos. É possível notar que as revistas nacionais ainda não atingiram presença significativa nos principais indexadores científicos, uma vez que nenhum grupo de periódicos pertencentes aos diferentes estratos da classificação Capes atinge alta presença nos indexadores (vide legenda), ou seja, mais de 80\% de periódicos presentes. Sobre este aspecto, destacamos que nossos periódicos só conseguem ter presença regular no Latindex, o que indica que ainda há muito a ser desenvolvido para que aumentemos nossa disseminação científica em âmbito Nacional, Ibero-americano e Internacional.

Também é possível notar, a partir dos resultados, que não há presença de periódicos de estrato inferior (B3, B4 e B5) nos indexadores Web of Science, Scopus e Scielo. Talvez uma das explicações para que isto ocorra seja o maior número de critérios de boas práticas que estes indexadores exigem para que os periódicos componham suas bases. Em contrapartida, outro resultado que chama a atenção é a elevada presença de periódicos de altos estratos (A2, B1 e B2) nos indexadores Latindex, Google Scholar e Spell:

Tabela 1: Presença das nos principais indexadores identificados na literatura (por estrato Qualis).

\begin{tabular}{c|c|c|c|c|c|c|c}
\hline Estrato & $\mathbf{A 2}$ & $\mathbf{B 1}$ & $\mathbf{B 2}$ & $\mathbf{B 3}$ & $\mathbf{B 4}$ & B5 & Total \\
\hline LATINDEX & 15 & 27 & 38 & 49 & 36 & 12 & 177 \\
\% & $88.24 \%$ & $90.00 \%$ & $90.48 \%$ & $70.00 \%$ & $33.64 \%$ & $31.58 \%$ & $58.22 \%$ \\
\hline $\boldsymbol{S C H O L A R}$ & 16 & 26 & 37 & 31 & 20 & 5 & 135 \\
\% & $94.12 \%$ & $86.67 \%$ & $88.10 \%$ & $44.29 \%$ & $18.69 \%$ & $13.16 \%$ & $44.41 \%$ \\
\hline SPELL & 16 & 23 & 37 & 29 & 3 & 1 & 109 \\
\% & $94.12 \%$ & $76.67 \%$ & $88.10 \%$ & $41.43 \%$ & $2.80 \%$ & $2.63 \%$ & $35.86 \%$ \\
\hline $\boldsymbol{D O A J}$ & 13 & 21 & 25 & 41 & 10 & 3 & 113 \\
\% & $76.47 \%$ & $70.00 \%$ & $59.52 \%$ & $58.57 \%$ & $9.35 \%$ & $7.89 \%$ & $37.17 \%$ \\
\hline $\boldsymbol{E B S C O}$ & 13 & 21 & 32 & 22 & 2 & 3 & 93 \\
\% & $76.47 \%$ & $70.00 \%$ & $76.19 \%$ & $31.43 \%$ & $1.87 \%$ & $7.89 \%$ & $30.59 \%$ \\
\hline
\end{tabular}


ROSA, Rodrigo Assunção; ROMANI-DIAS, Marcello

"Periódicos científicos brasileiros a partir de seus indexadores:cenário atual e perspectivas futuras para a administração, contabilidade e turismo"

\begin{tabular}{c|ccccccc}
\cline { 3 - 7 } REDALYC & 14 & 13 & 17 & 3 & 1 & 0 & 48 \\
$\boldsymbol{\%}$ & $82.35 \%$ & $43.33 \%$ & $40.48 \%$ & $4.29 \%$ & $0.93 \%$ & $0.00 \%$ & $15.79 \%$ \\
\hline PROQUEST & 9 & 9 & 11 & 10 & 1 & 1 & 41 \\
$\boldsymbol{\%}$ & $52.94 \%$ & $30.00 \%$ & $26.19 \%$ & $14.29 \%$ & $0.93 \%$ & $2.63 \%$ & $13.49 \%$ \\
\hline SCIELO & 12 & 5 & 1 & 0 & 0 & 0 & 18 \\
$\boldsymbol{\%}$ & $70.59 \%$ & $16.67 \%$ & $2.38 \%$ & $0.00 \%$ & $0.00 \%$ & $0.00 \%$ & $5.92 \%$ \\
\hline SCOPUS & 6 & 5 & 2 & 0 & 0 & 0 & 13 \\
$\%$ & $35.29 \%$ & $16.67 \%$ & $4.76 \%$ & $0.00 \%$ & $0.00 \%$ & $0.00 \%$ & $4.28 \%$ \\
\hline $\boldsymbol{W O S}$ & 2 & 1 & 0 & 0 & 0 & 0 & 3 \\
$\%$ & $11.76 \%$ & $3.33 \%$ & $0.00 \%$ & $0.00 \%$ & $0.00 \%$ & $0.00 \%$ & $0.99 \%$ \\
\hline
\end{tabular}

Legenda de Presença dos Periódicos nos Indexadores :

Acima de 80\% - Presença Alta

Entre 50.01 e 80\% - Presença Regular

Entre 20 e 50\% - Presença Baixa

Abaixo de 20\% - Presença Muito Baixa

$0 \%$ - Inexistente

Há também resultados pertinentes aos 188 indexadores identificadas a partir dos websites dos periódicos nacionais de modo geral. Identificamos que a maioria dos indexadores (140 no total) foram relatados por apenas uma a cinco revistas. Em outras palavras, somente aproximadamente $25 \%$ dos indexadores são relatados por mais de seis (dos 304) periódicos que compõem a população. Este resultado parece indicar que são poucos os indicadores que realmente indexam um número substancial de revistas nacionais, e que entre eles destacam-se, além de alguns dos dez indexadores que já analisamos anteriormente, indexadores como Sumários.org, Diadorim, Acesso Livre e Ulrich's. Todos estes indexadores possuem mais de 30 revistas nacionais da área indexadas e, por esta razão, podem tornar-se critérios adicionais de classificação do Qualis-Periódicos no futuro, dado que possuem amplo potencial de disseminação da publicação científica.

Além disso, notamos que, em média, tanto as revistas pertencentes ao estrato B1 quanto as pertencentes ao estrato B3 têm presença em um número maior de indexadores do tipo geral, quando comparadas às revistas do estrato A2. Este resultado, aparentemente contra intuitivo, parece indicar que não é suficiente que a revista tenha um maior número de indexadores para melhorar sua qualidade, sendo necessário, portanto, que os periódicos conheçam os principais indexadores científicos, estejam estes ou não atrelados ao QualisPeriódicos, o que também é corroborado pela alta presença de periódicos de estratos superiores nos principais indexadores e também nos indexadores que realizam o cálculo de fator de impacto. Adicionalmente, notamos que os periódicos dos estratos B4 e principalmente B5 possuem uma presença média significativamente menor nos indexadores gerais quando comparados aos periódicos de estratos mais elevados - haja visto que nenhum 
periódico B4 ou B5 estão presentes em mais do que 11 indexadores - o que parece indicar que, se por um lado é necessário conhecer os indexadores para buscar a qualidade na publicação, por outro, há também um número mínimo de indexadores de que uma revista científica deve, preferencialmente, fazer parte para atender aos critérios e galgar posições na classificação da Capes.

\section{IMPLICAÇÕES PARA A PUBLICAÇÃO CIENTÍFICA BRASILEIRA}

A partir da última avaliação realizada, a Capes (2017) anunciou mudanças substanciais nos critérios de avaliação de periódicos. Além da consolidação da base Spell como critério de qualidade de periódicos, destacamos uma mudança especial: somente serão considerados periódicos científicos, mesmo em estratos menores (B4 e B5) aqueles que fizerem parte de indexadores como Doaj, Ebsco e Redalyc. Trata-se de uma mudança que funcionará como um ponto de inflexão na trajetória da produção científica brasileira.

Com base nos resultados que alcançamos entendemos que se tal mudança fosse trazida para os dias atuais diversos periódicos nacionais da área seriam rebaixados ao estrato C. Como consequência disto, o critério poderá trazer prejuízos para os periódicos, entre os quais destacamos a abrupta exclusão destes, visto que necessitam de recursos e incentivos para sua consolidação e que, neste cenário, estariam sendo penalizadas em decorrência desta mudança.

Compreendemos que não precisamos restringir a escolha dos periódicos somente entre os indexadores pretendidos pela Capes. Indexadores como Google Scholar e Latindex, por exemplo, exigem que os periódicos estabeleçam determinadas boas práticas e, no entanto, são excluídos do atual critério de classificação. A inclusão destes indexadores pode trazer oportunidades de melhorias na gestão dos periódicos que estão em estratos mais baixos e, desse modo, permitindo sua sobrevivência e dando tempo para que estes periódicos amadureçam para indexadores de transição (Ebsco, Proquest, Redalyc e Doaj) e, posteriormente, para indexadores consolidados (WOS, Scopus e Scielo).

Acreditamos que a Capes pode repensar sua forma de avaliação não levando em consideração apenas o fator de impacto das revistas, mas também um conjunto maior de parâmetros relacionados ao periódico, como: se este realiza publicações bilíngues ou inglês e se está presente em um conjunto de indexadores considerados relevantes pela comunidade acadêmica, tais como os identificados nesta pesquisa. Este conjunto de medidas também pode, de algum modo, melhorar o processo de internacionalização, uma vez que as revistas precisam umas das outras para melhorar seus impactos nas principais bases científicas, visto 
que estas medidas são dependentes das relações de citação estabelecidas entre os periódicos (Diniz, 2017).

Observamos a partir da literatura e dos dados que há indexadores centrais que devem ser considerados, tendo em vista, por exemplo, sua cobertura, existência de busca integrada e cálculo de fator de impacto. Todavia, conforme destaca Kimura et al (2014), devemos compreender que os periódicos adotam posicionamentos distintos, e que cada um deve seguir seus objetivos e posicionamento, visto que nem todo o periódico almeja, por exemplo, disseminação internacional.

Independentemente do posicionamento do periódico e dada a inevitabilidade da indexação como critério para que o periódico seja considerado científico pela classificação da Capes, consideramos que as revistas devem refletir a respeito das possibilidades de indexação para a melhoria de suas práticas de gestão científica e editorial. Além disso, nossa análise também demonstra que ao pensar em indexadores de consolidação e na utilização de opções de idiomas como bilíngue e inglês, determinadas revistas podem alcançar melhor difusão internacional. No entanto, consideramos que este movimento de internacionalização não deve ser pensado de forma individual por cada periódico, mas sim de maneira conjunta e a partir de apoios institucionais (Diniz, 2017). Também entendemos que as revistas que já estão consolidadas em estratos superiores devem pensar conjuntamente em alternativas para aumentar sua disseminação em escala global, diante da fragilidade de imagem que podem possuir internacionalmente (Alcadipani, 2017). Por fim, defendemos que as revistas brasileiras pertencentes aos estratos superiores pela classificação da Capes possuem a responsabilidade de serem pioneiras na disseminação de nosso conhecimento em Administração, Contabilidade e Turismo.

\section{REFERÊNCIAS}

ALCADIPANI, Rafael. Periódicos brasileiros em inglês: a mímica do publish or perish "global". Revista de Administração de Empresas, v. 57, n. 4, p. 405-411, 2017.

BERTERO, Carlos Osmar et al. Os desafios da produção de conhecimento em administração no Brasil. Cadernos EBAPE. BR, v. 11, n. 1, 2013.

COORDENAÇÃO DE APERFEIÇOAMENTO DE PESSOAL DE NÍVEL SUPERIOR. Relatório quadrienal 2017. Disponível em: $<$ http://www.capes.gov.br/images/documentos/Relatorios_quadrienal_2017/Administração_v 2.pdf>. Acesso em: 29 set. 2017.

COLLAZO-REYES, Francisco. Growth of the number of indexed journals of Latin America and the Caribbean: the effect on the impact of each country. Scientometrics, v. 98, n. 1, p. 197-209, 2014. 
DINIZ, Eduardo H. Periódicos brasileiros da área de Administração no contexto de internacionalização da produção científica. Revista de Administração de Empresas, v. 57, n. 4, p. 357-364, 2017.

FARIAS, SALOMÃO ALENCAR DE. Internacionalização dos periódicos brasileiros. Revista de Administração de Empresas, v. 57, n. 4, p. 401-404, 2017.

HARZING, Anne-Wil; ALAKANGAS, Satu. Google Scholar, Scopus and the Web of Science: a longitudinal and cross-disciplinary comparison. Scientometrics, v. 106, n. 2, p. 787-804, 2016.

KIMURA, Herbert et al. O processo de internacionalização de periódicos nacionais. RACRevista de Administração Contemporânea, v. 18, n. 6, 2014.

OLIVEIRA, Carla Cristina Vieira de; CENDÓN, Beatriz Valadares; CIRINO, Sergio Dias. Aspectos estruturais considerados nos estudos de qualidade dos periódicos científicos. Pesquisa Brasileira em Ciência da Informação e Biblioteconomia, v. 12, n. 1, 2017.

ROZEMBLUM, Cecilia et al. Calidad editorial y calidad científica en los parámetros para inclusión de revistas científicas en bases de datos en Acceso Abierto y comerciales. Palabra clave, v. 4, n. 2, p. 64-80, 2015.

SANDES-GUIMARÃES, Luisa Veras de; DINIZ, Eduardo H. Gestão de periódicos científicos: estudo de casos em revistas da área de Administração. 2014.

TRZESNIAK, Piotr. Qualis in four quarters: history and suggestions for the Administration, Accounting and Tourism area. Revista Contabilidade \& Finanças, v. 27, n. 72, p. 279-290, 2016. 